\title{
Observations of artificial and natural optical emissions at the HAARP facility
}

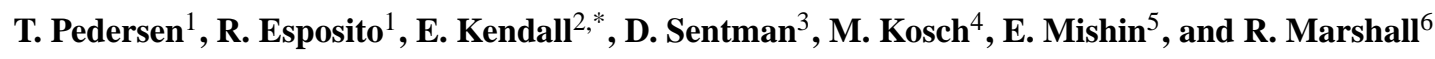 \\ *formerly: Gerken \\ ${ }^{1}$ Space Vehicles Directorate, Air Force Research Laboratory, AFRL/VSBXI, 29 Randolph Rd., Hanscom AFB, MA 01731, \\ USA \\ ${ }^{2}$ SRI International, 333 Ravenswood Avenue, Menlo Park CA 94025, USA \\ ${ }^{3}$ Geophysical Institute, University of Alaska, Fairbanks, 903 Koyukuk Drive, Fairbanks, AK 99775, USA \\ ${ }^{4}$ Department of Communications Systems, Lancaster University, InfoLab21, Lancaster University, Lancaster LA1 4WA UK \\ ${ }^{5}$ Institute for Scientific Research, Boston College, AFRL/VSBXI, 29 Randolph Rd., Hanscom AFB, MA 01731, USA \\ ${ }^{6}$ STAR Lab, Stanford University, 350 Serra Mall, Stanford, CA 94305, USA
}

Received: 15 January 2007 - Revised: 23 July 2007 - Accepted: 25 July 2007 - Published: 28 May 2008

\begin{abstract}
Extensive optical observations have been carried out at the High Frequency Active Auroral Research Program (HAARP) ionospheric heating facility since it began operations in 1999. A number of modern optical diagnostic instruments are hosted at remote sites as well as the main transmitter facility, which has recently been expanded from the initial $960 \mathrm{~kW}$ prototype configuration to its full 3.6 MW design capability. Upgrades to optical diagnostics have allowed a number of interesting new observations to be made at the $960 \mathrm{~kW}$ power level since 2004. Systematic beam-swinging experiments generating quantifiable levels of optical emission at various regions in the sky for the first time clearly show that emission intensity is very sensitive to distance from the magnetic zenith, and drops off rapidly at about $15^{\circ}$ zenith angle in directions other than magnetic south. High temporal resolution measurements of emissions in the $557.7 \mathrm{~nm}$ green line at start-up and in short transmitter pulses demonstrate that localized irregularities are preferentially excited in the initial seconds of heating, with evolution into a more homogenous spot occurring over a period of about $1 \mathrm{~min}$. Highquality emission altitude profiles at both 630.0 and $557.7 \mathrm{~nm}$ have recently been isolated from side-looking data, spanning an altitude extent of over $200 \mathrm{~km}$, which has allowed determination of the effective lifetime of $\mathrm{O}\left({ }^{1} \mathrm{D}\right)$ over an unprecedented altitude range. An innovative automated remote imager network utilizing low-cost mirror optics has been designed and deployed to make such measurements routinely. Observations of natural optical emissions at the site have revealed the common presence of highly structured but faint co-rotating subauroral precipitation that acts to suppress ex-
\end{abstract}

Correspondence to: T. Pedersen

(no email available) citation of artificial $\mathrm{F}$ region optical emissions in areas of active precipitation. The observed spatial modulation of artificial optical emissions by structured precipitation is consistent with localized absorption of HF waves in the ionospheric D layer enhanced by the energetic particle precipitation.

Keywords. Atmospheric composition and structure (Airglow and aurora) - Ionosphere (Active experiments; Instruments and techniques)

\section{Introduction}

The High Frequency Active Auroral Research Program (HAARP) has operated an ionospheric heating facility in Gakona, Alaska $\left(62.4^{\circ} \mathrm{N} 145^{\circ} \mathrm{W}, \sim 63^{\circ}\right.$ magnetic) since 1999. The main HF transmitter, or Ionospheric Research Instrument (IRI), in initial prototype configuration consisted of an array of $6 \times 8$ crossed dipoles providing $960 \mathrm{~kW}$ total power, with nominal effective radiated power (ERP) ranging from $10.8 \mathrm{MW}$ at $2.8 \mathrm{MHz}$ to $260 \mathrm{MW}$ at $10 \mathrm{MHz}$. The transmitter array has recently been expanded to $15 \times 12$ elements producing a total power of $3.6 \mathrm{MW}$, corresponding to nominal ERP of $260 \mathrm{MW}$ at $2.8 \mathrm{MHz}$ and $4400 \mathrm{MW}$ at $10 \mathrm{MHz}$. The IRI beam can be steered by electronic phasing of the antenna elements to any point within $30^{\circ}$ of zenith, although movements of more than $15^{\circ}$ from a pre-tuned position require several seconds for re-tuning.

Diagnostic instrumentation has been an essential part of the HAARP facility since its inception, and a number of optical instruments have been deployed and operated at the site as part of the facility's permanent diagnostics, with others operated at remote locations. The current optical diagnostics

Published by Copernicus Publications on behalf of the European Geosciences Union. 
on site consist of the HAARP imager, the HAARP telescope, and the HAARP photometer. The HAARP imager is a telecentric system utilizing $3.5^{\prime \prime}$ narrow-band $(2 \mathrm{~nm})$ filters feeding a recently upgraded 1 " thermo-electrically cooled bare CCD camera. It can be operated with either a fish-eye lens for all-sky coverage or placed on a mount and equipped with a $16^{\circ}$ lens for higher resolution observations of optical emissions within the HAARP beam. The HAARP telescope consists of an $8^{\prime \prime}$ diameter refractor telescope with $3^{\prime \prime}$ filters imaged by a $1 / 2^{\prime \prime}$ cooled bare CCD camera. It includes a second similar camera with approximately $14^{\circ}$ field of view for context. The HAARP photometer has 2 dedicated channels for observation of the $630.0 \mathrm{~nm}$ and $557.7 \mathrm{~nm}$ oxygen lines, plus a third channel equipped with a filter wheel, which can be operated at a single wavelength or scanned through all of the filters in rapid succession. A recent addition to the HAARP optical diagnostic suite is the first of a network of remote imaging systems, to be discussed in detail in a later section.

The first optical observations at HAARP were in March 1999 , when $630 \mathrm{~nm}$ red line enhancements of $\sim 150$ Rayleigh (R) were seen during vertical heating, although with extremely poor sensitivity due to an observation mode optimized for natural aurora (Pedersen and Carlson, 2001). Significant emissions were not seen again until March 2001, also during vertical heating, although in both these early experiments there were indications of enhanced emissions in the magnetic zenith. Experiments to direct the beam into the magnetic zenith were first carried out in early February 2002, and over the course of the campaign a large number of observations were made of the localized bright spot about $7^{\circ}$ in diameter which appeared in the magnetic zenith under a wide range of conditions (Pedersen et al., 2003). The 2003 campaign featured the first attempt to stimulate electron gyroharmonic resonances, but poor weather and active aurora limited useful observations to only a few minutes. In the winter of 2004, good conditions were encountered and a number of measurements made at the 2nd and 3rd gyroharmonics (Djuth et al., 2005; Kosch et al., 2005), which allowed detectable emissions in both 630.0 and $557.7 \mathrm{~nm}$ lines to be produced even at the extremely low ERP resulting from the broad beam at low frequencies near 2.8 MHz. The 2004 campaign also marked a turning point in diagnostics, as improved imagers came into permanent use at this time and the telescope was able to routinely image structure within the airglow spot.

In the sections that follow, we present and discuss observations made since 2004, when a variety of new and interesting features in both artificial and natural optical emissions have been observed, many resulting from the combination of improved optical instruments and the highly efficient 2nd gyroharmonic resonance. Many of these observations are unique, depending upon capabilities or modes not available at other ionospheric heaters or resulting from interactions with the unique local environment.

\section{Observations of artificial optical emissions}

\subsection{Beam swinging experiments}

One of the unique capabilities of the HAARP transmitter is its ability to be repositioned over a relatively wide range of angles. This capability was employed in the first observations of the magnetic zenith spot at HAARP, in the form of a conical scan at $15^{\circ}$ zenith angle (Pedersen et al., 2003). These early measurements at HAARP showed indications of faint emissions from beam positions other than magnetic zenith, and several cases of optical emissions from vertical heating were known from HAARP prior to active stimulation of the magnetic zenith effect. Until improved imagers were deployed in the last few years, however, the threshold for detection of emissions at other beam positions generally remained too high.

On 6 February 2005, a beam swinging experiment was conducted specifically to investigate the dependence of launch angle on optical emission production efficiency. The transmitter beam was slewed between magnetic zenith $\left(204^{\circ}\right.$ az. $15^{\circ}$ zen., used as a control,) and a variety of positions including the vertical and $15^{\circ}$ and $30^{\circ}$ off zenith in each of the cardinal magnetic directions. The beam alternated between $2 \mathrm{~min}$ in other positions and $2 \mathrm{~min}$ in the magnetic zenith, with $30 \mathrm{~s}$ allocated for repositioning between positions to round out a $5 \mathrm{~min}$ cycle. The experiment was run in the decaying twilight ionosphere, which allowed for frequencies from $4.3 \mathrm{MHz}$ down to $2.8 \mathrm{MHz}$ near the 2nd gyroharmonic to be utilized. Figure 1 shows sample $630 \mathrm{~nm}$ red-line images from this experiment, with power contours for the $2.83 \mathrm{MHz}$ beam superimposed on the all-sky images, which in this experiment were oriented to magnetic coordinates. The lower left side of each image is contaminated with twilight, and the natural aurora can be seen on the far northern horizon. The position of the magnetic zenith is marked with an "X".

In the first three cases the beam center is far from the magnetic zenith, which is on or outside the $10 \%$ power contour. The artificial emissions can be seen to be of the diffuse beam-filling type, representing enhancements of a few tens of Rayleigh above the background. Rather than being distributed throughout the beam, however, the emissions are most intense in the sector of the beam toward magnetic zenith. Emissions reach a maximum approximately midway between the magnetic zenith and the beam center. The lack of significant emissions in the beam center in spite of the power peaking there suggests that either refraction of the rays back toward the earth has prevented the waves from reaching a resonant altitude, or that the aspect-angle dependence of the optical production mechanism dominates over the increasing gradient in ERP toward the center of the beam.

The fourth frame in the figure shows optical emissions produced when the beam was pointed $30^{\circ}$ from zenith toward magnetic south, or $15^{\circ}$ beyond the magnetic zenith. The 


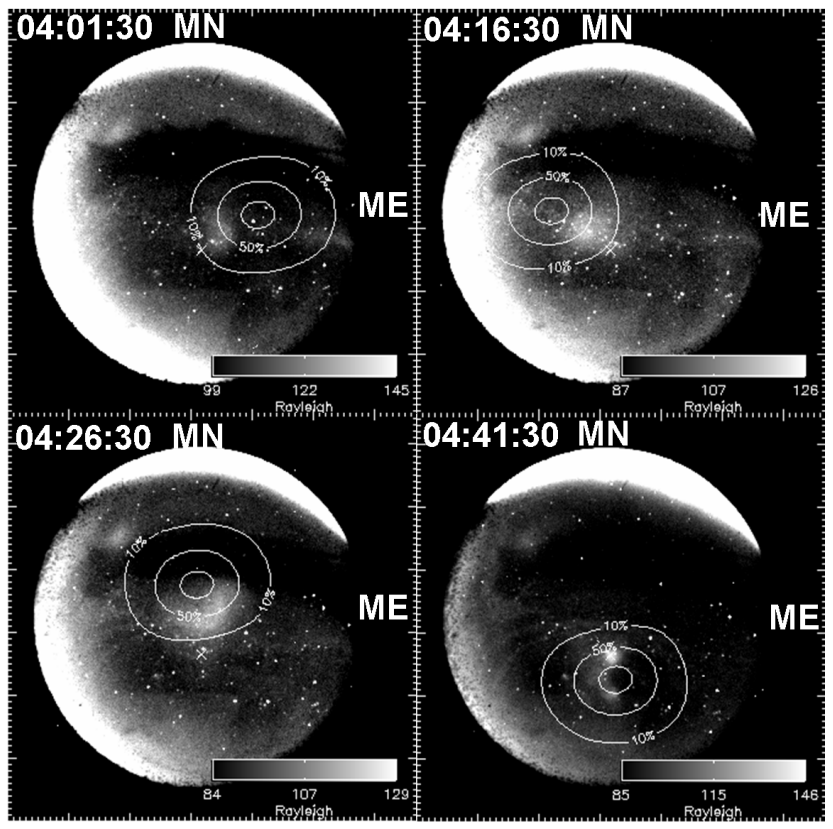

Fig. 1. $630.0 \mathrm{~nm}$ emissions produced at various angles off vertical on 6 February 2005: $30^{\circ}$ toward magnetic east (upper left), $30^{\circ}$ toward magnetic west (upper right), $15^{\circ}$ toward magnetic north (lower left), and $30^{\circ}$ toward magnetic south (lower right). Beam contours for 10,50 , and $90 \%$ of maximum power are shown, and the position of magnetic zenith is marked with an "X". Twilight forms a broad band at lower left in each image, while the distant aurora can be seen at top.

magnetic zenith sits on the $50 \%$ contour at this point, and the typical magnetic zenith spot is stimulated and produces approximately $50 \mathrm{R}$ of emissions. Secondary enhancements including a bright spot on the $90 \%$ contour on the far side of the beam can also be seen, indicating that waves directed more than $30^{\circ}$ from zenith have reached some resonant altitude. The beam center, however, is lacking significant emissions, although in this case the irregular nature of the emissions suggest that large-scale irregularities could be present, ducting the radio waves along paths not possible in a smooth ionosphere. The patchy nature of the emissions observed on this occasion, which was toward the end of the experiment, may result from penetration of the transmitter beam through regions of lower density as the average ionospheric critical frequency approached the transmitter frequency, as discussed by Kosch et al. (2005).

Line plots of calibrated emission intensity through these images from geographic zenith toward the beam center are shown in Fig. 2, along with transmitter beam power. These provide additional insight into the spatial dependence of the emissions, and reveal a significant asymmetry between the magnetic meridian and the direction perpendicular to it. The upper left panel shows emissions in the magnetic east rising along with beam power up to about $7^{\circ}$ from zenith, after
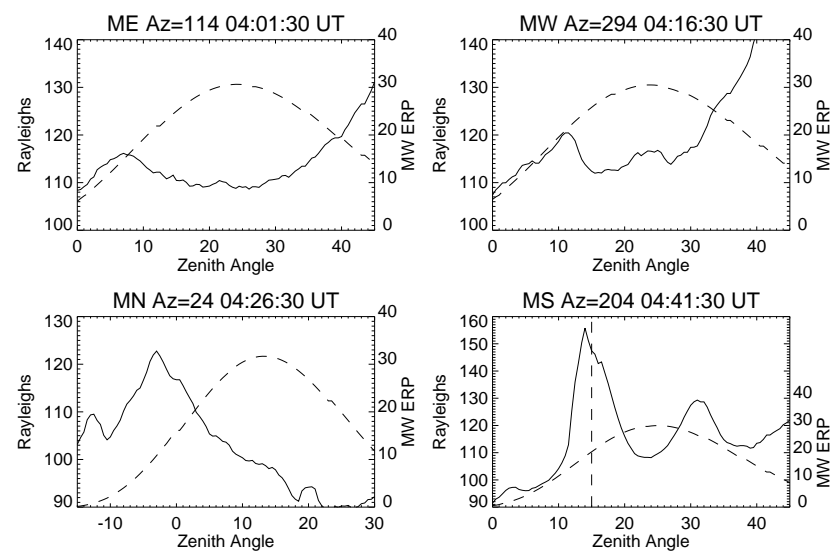

Fig. 2. Line profiles of $630 \mathrm{~nm}$ emission intensity (solid) and HAARP beam ERP (dashed) for the images shown in Fig. 1. Profiles are along the cardinal magnetic directions in the geographic azimuths as indicated at the top of each panel. Increases in intensity beyond $30^{\circ}$ zenith angle are due to the natural sky background.

which they drop off rapidly to background levels in spite of beam power rising threefold over the next $20^{\circ}$. Note that the large increase in optical intensity beyond $30^{\circ}$ zenith angle in each of the profiles in this figure results from the natural optical background increasing at lower elevation angles and does not reflect any artificial effects. The upper right panel for magnetic west shows the same basic behavior as magnetic east, with emissions rising along with beam power for the first $\sim 10^{\circ}$ before dropping precipitously to background levels. In both cases perpendicular to the magnetic meridian the intensity has dropped to background levels by about $15^{\circ}$ zenith angle, regardless of the beam power.

Along the magnetic meridian, however, the behavior is significantly different. The lower left panel shows beam power directed at $15^{\circ}$ magnetic north, but the emissions peak approximately $20^{\circ}$ southward, just south of zenith, and drop off sharply to reach background levels near $20^{\circ}$. The magnetic zenith enhancement is also clearly visible near $-13^{\circ}$ zenith angle in spite of beam power approaching zero there. The beam directed at $30^{\circ}$ magnetic south, at lower right, produces a large enhancement in the magnetic zenith at $15^{\circ}$ zenith angle, and a secondary enhancement near $30^{\circ}$, which appears to result from the irregular ionospheric structure present at this time rather than being a permanent spatial feature. Taken as a whole, these data indicate production efficiency dropping off rapidly by $\sim 15^{\circ}$ toward the $\mathrm{E}$ and $\mathrm{W}$. In the meridian, efficiency drops equally rapidly toward the $\mathrm{N}$, but is significant from vertical through and well beyond MZ. A full ray-tracing analysis through a realistic 4-D ionospheric reconstruction will be required to determine whether the observed cut-offs result from propagation conditions preventing the transmitter beam from reaching the interaction altitude or are a feature of the ionospheric interaction mechanism. 


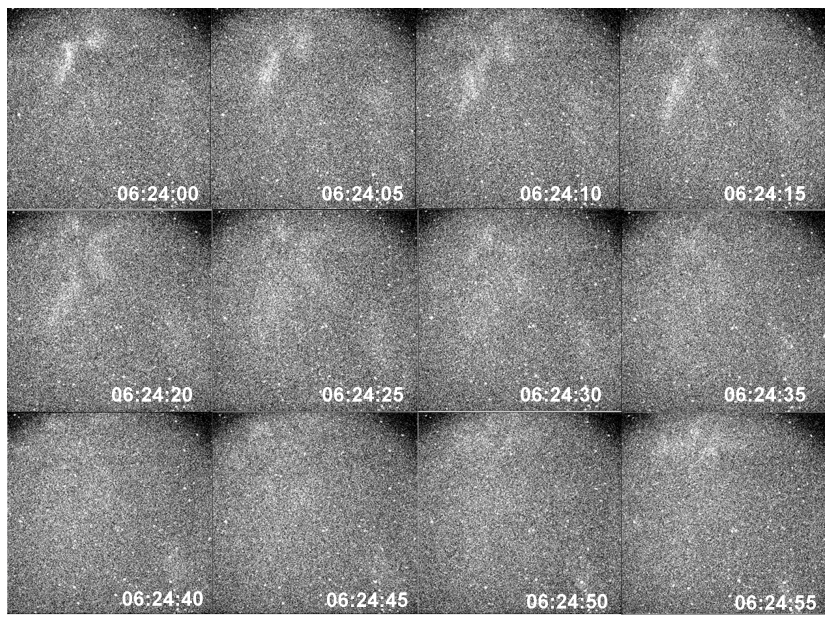

Fig. 3. $557.7 \mathrm{~nm}$ emissions in the magnetic zenith imaged at $5 \mathrm{~s}$ intervals during the first minute of a 2 min transmitter pulse on 25 March 2006. Each frame is approximately $9^{\circ}$ across. Time stamps refer to the start time for each $5 \mathrm{~s}$ exposure.

\subsection{High temporal resolution imaging}

Optical imaging at HAARP had previously been limited to frame rates of $\sim 10$ s or greater due to sensitivity, readout and housekeeping requirements on the various imager systems, but in light of some 2005 results showing beam-filling green line emissions collapsing into the magnetic zenith spot during the first $\sim 1 \mathrm{~min}$ of heating (Kosch et al., 2007a), which also suggested an possible explanation for the green line onset delay first observed in 2002 (Pedersen et al., 2003), a concentrated effort to image the development of the emissions at higher time resolution was made in the most recent 2006 optical campaign. An electron multiplication charge-coupled device (EM-CCD) camera was employed for the first time, and conventional CCD cameras were operated with minimal exposure time at multiple wavelengths to increase the overall temporal resolution. These diagnostic techniques were combined with a pulsed transmitter mode to investigate initial conditions and observe the evolution of the HAARP spot.

Figure 3 shows a 1-min sequence of narrow-field $557.7 \mathrm{~nm}$ images taken at $5 \mathrm{~s}$ intervals during the first minute of a 2min transmitter pulse at $2.83 \mathrm{MHz}$, near the 2 nd harmonic of the electron gyrofrequency. The $\sim 9^{\circ}$ field of view corresponds to $\sim 40 \mathrm{~km}$ horizontal distance at $250 \mathrm{~km}$ altitude. In the first frame, two irregular localized regions approximately $5-10 \mathrm{~km}$ in size light up. Over the next $20 \mathrm{~s}$ or so, these structures move steadily while diffusing out, until at $30 \mathrm{~s}$ after turn-on the region is relatively diffuse, although new, less distinct structure continues to appear and drift across the field of view, a motion which is much more readily apparent in movies of the image data. As the typical diameter of the magnetic zenith spot is on the order of the size of these images, the overall increase in unstructured $557.7 \mathrm{~nm}$ emis- sions within the spot relative to the background sky is not readily apparent from this data. Significant optical structure within the magnetic zenith spot is common at HAARP in high-resolution $557.7 \mathrm{~nm}$ images (see Djuth et al., 2005, for an example), but with integration times of up to $30 \mathrm{~s}$ required, the initial dynamics of the small-scale structures were not able to be determined, beyond the fact that the structure generally moves steadily across the spot. It is clear from the new high time resolution data, however, that localized small-scale structures are preferentially excited in the first few seconds of heating, and that the overall character of the emissions becomes more diffuse as time progresses.

In addition to high temporal resolution measurements of the leading edge of standard multiple-minute transmitter pulses, a short-pulse mode specifically for higher-speed optical observations was also devised and run during the March 2006 campaign. Figure 4 shows such a customized transmitter pulse and exposure sequence employed on 30 March 2006. The $5 \mathrm{~min}$ O-mode sequence was run at a nominal ERP of $11 \mathrm{MW}$ and a frequency of $2.81 \mathrm{MHz}$ targeting the 2nd harmonic of the electron gyrofrequency at an altitude of $\sim 250 \mathrm{~km}$. The sequence started with 1 min of transmitter off, followed by 510 -s periods consisting of $2 \mathrm{~s}$ on and $8 \mathrm{~s}$ off to allow diagnostic measurements while minimizing effects on the ionosphere. The second minute was finished off by a $10 \mathrm{~s}$ on period that was certain to provide adequate exposure time if no signals rose above the detection threshold during the $2 \mathrm{~s}$ pulses. Another minute of off was allowed before the main pulse of 2 min of full power completed the sequence. Three cameras (1 all-sky and 2 narrow-field systems observing at 557.7 and $630.0 \mathrm{~nm}$ ) were operated according to the exposure sequence represented by the dashed line near the top of the figure. The systems were synchronized to the transmitter to expose during the $2 \mathrm{~s}$ on pulses and take dark frames during the $8 \mathrm{~s}$ off periods, with longer exposures of up to $8 \mathrm{~s}$ occurring later in each minute.

This mode produced detectable green and red emissions during several of the initial $2 \mathrm{~s}$ pulses. The first pulse, beginning at 06:01:00 UT, showed no detectable emissions, but the second pulse $10 \mathrm{~s}$ later showed significant green line emissions in two small localized regions. These same regions lit up again on each successive pulse, and again during the first few exposures in the long power-on sequence before the emissions gradually diffused out into the normal spot similar to that seen in the $5 \mathrm{~s}$ resolution images described earlier. It is interesting to note that, unlike the continuous pulse experiment shown in Fig. 3, the irregular regions remained unchanged for the greater part of a minute, diffusing out only during the extended $2 \mathrm{~min}$ on period. These initial results, especially the lack of detectable emission on the first pulse, suggest the possibility that the localized bright spots are created by the transmitter itself during the first $2 \mathrm{~s}$ pulse which were then able to absorb sufficient energy from the beam to excite optical emissions on subsequent pulses. However, it is not clear how such large $(5-10 \mathrm{~km})$ irregularities could be 


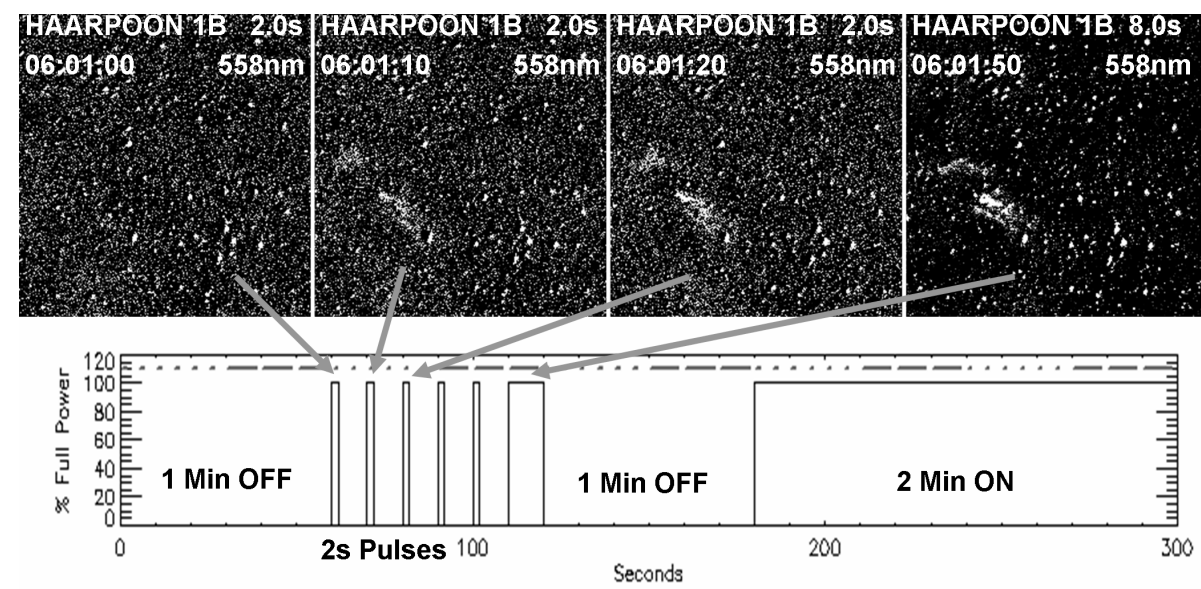

Fig. 4. Background-subtracted narrow-field $\left(\sim 15^{\circ}\right.$ FOV) green line images from the magnetic zenith taken during short transmitter pulses on 30 March 2006. The bottom panel shows the 5-min transmitter power modulation scheme (black line) and the camera exposure sequence (dashed gray line near top of panel), which repeated every minute.

created in such a short time, so some other explanation for their absence during the initial pulse may be required. The $5 \mathrm{~s}$ data in Fig. 3 are of insufficient temporal resolution to investigate irregularity formation in the first few seconds, but do clearly show a gradual diffusion and reduction in structure during the extended on period. A complicating factor for the observations in Fig. 4, however, is the presence of highly structured subauroral precipitation, of the type discussed in more detail in a later section, in the area during the experiment, which may have influenced the structure observed.

\subsection{Side looking imaging and altitude profiles}

Side-looking observations have been attempted in conjunction with HAARP heating campaigns from several remote locations since the original observations of artificial optical emissions in 1999, and have been an integral part of artificial optical emission measurements at the EISCAT HEATING facility as well (Brandstrom et al., 1999; Gustavsson et al., 2001; Brandstrom, 2003). Useful side-looking images of HAARP artificial emissions have been available from Poker Flat, Alaska, $325 \mathrm{~km}$ from the HAARP site, since 2002. These show the magnetic zenith spot to be roughly spherical, with a similar extent in both altitude and horizontal diameter. Significant field-aligned structure is also observed moving through the spot, presumably with the $\boldsymbol{E} \times \boldsymbol{B}$ drift imposed by the background electric field, and represents the projection along the magnetic field of the moving irregular structure seen from below by cameras at the HAARP site. The first useful altitude profiles from the $557.7 \mathrm{~nm}$ green line were only successfully extracted recently, from data taken during the February 2005 campaign (Kosch et al., 2007a). Additionally, since 2004, all-sky images from the HAARP facility have been able to capture altitude profiles of artificial optical emissions created by the HIPAS facility near Fair- banks, Alaska, providing a second source of altitude information on artificial optical emissions.

Figure 5 shows an example of HAARP altitude profiles extracted from the Poker Flat remote imaging data. Optical images taken at 557.7 and $630.0 \mathrm{~nm}$ approximately every $20 \mathrm{~s}$ have been averaged horizontally across the projection of the magnetic field line passing through the HAARP transmitter, warped to a common grid of time vs. altitude, and displayed as a composite-color keogram image. Stars were removed with a median filter, and flat-fielding was performed to eliminate instrumental reductions in intensity away from the image centers. Raw pixel values were converted to Rayleighs by assuming a spherical form for the emission spot and comparing intensities with those from the fully calibrated HAARP imager viewing the emissions from below. Contours from hand-scaled ionosonde profiles were then superimposed on the keogram. The optical emissions can be seen to extend over an altitude range of up to $200 \mathrm{~km}$ and are most intense at an altitude below the reflection $(2.85 \mathrm{MHz})$ altitude but near or just below the altitude of the upper hybrid resonance (labeled "fUH"). The emissions noticeably continue well after all reflection at the transmitter frequency has been lost ( 04:05 UT) but die out as soon as the upper hybrid frequency is passed just after 04:30 UT, consistent with Kosch et al. (2005).

The extended lifetime of the red line emissions can be clearly seen by the change in hue to red after the end of each $1 \mathrm{~min}$ pulse, and the reduction in lifetime with altitude is also apparent in the change toward redder hues (and therefore relatively stronger $630.0 \mathrm{~nm}$ emissions) at higher altitudes. The altitude dependence of the red line effective emission lifetime $\tau$ can be examined in more quantitative terms by fitting emission intensities from individual pulses to a simple model allowing for the exponential dependence on the lifetime (cf. 


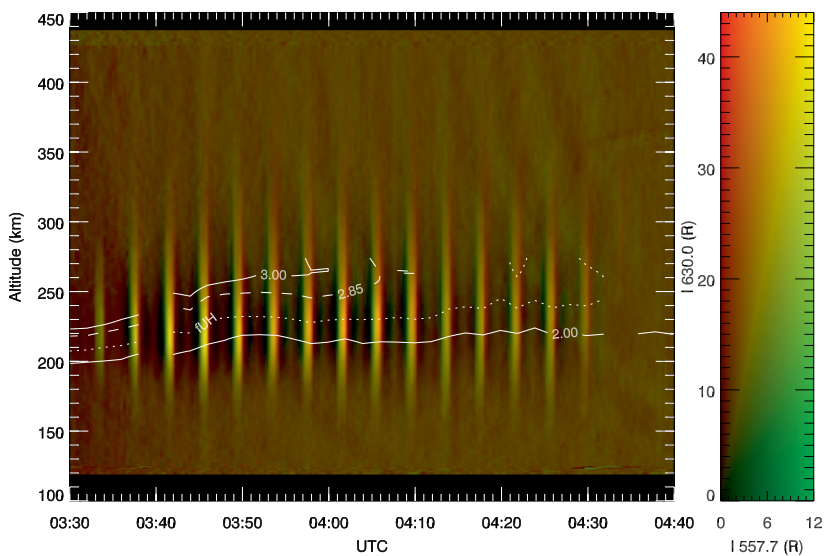

Fig. 5. Calibrated composite color profiles (red=630.0 nm, green $=557.7 \mathrm{~nm}$ ) of emission intensity vs. altitude along the HAARP field line collected on 4 February 2005 from Poker Flat, Alaska, $\sim 325 \mathrm{~km}$ from the HAARP site. Plasma frequency contours from the HAARP ionosonde, including the transmitter frequency of $2.85 \mathrm{MHz}$ and the upper hybrid frequency matching the transmitter frequency ("fUH") have been superimposed on the keogram. The scale at right indicates color as a function of red and green intensities.

Bernhardt et al., 1989; Gustavsson et al., 2001), varying peak saturation intensities in each transmitter pulse, and the integration and sampling times of the CCD cameras. This analysis provides, for the first time ever at HAARP, estimates of the $\mathrm{O}\left({ }^{1} \mathrm{D}\right)$ effective emission lifetime as a function of altitude. In the side-looking data from 4 February 2005 presented in Fig. 5, detectable emissions were present over an altitude range of approximately $200 \mathrm{~km}$, from about $150 \mathrm{~km}$ to $350 \mathrm{~km}$. Of this range, the analysis was able to retrieve effective lifetime estimates for altitudes between 190 and $350 \mathrm{~km}$. Outside of this range the algorithm failed to converge due to low signal level. Figure 6 shows the results of the analysis. Error bars represent the ratio of the root-meansquare (RMS) deviation between the data and model and the RMS intensity of artificial optical emissions above the ambient background, expressed as a fraction of the best-fit effective lifetime, i.e., $\Delta \tau=\tau \times\left(\operatorname{RMS}\left(I_{6300}-M\right)\right) /\left(\operatorname{RMS}\left(I_{6300}\right)\right)$, where $I_{6300}$ is the measured artificial enhancement in intensity above the sky background and $M$ is the best-fit modeled intensity. The effective lifetime is found to be just below $20 \mathrm{~s}$ at $190 \mathrm{~km}$ altitude and to rise to over $90 \mathrm{~s}$ at an altitude of $350 \mathrm{~km}$. These results can be compared with previously published altitude-resolved effective lifetime measurements from ionospheric heating, such as Bernhardt et al. (2000) and the tomography study of Gustavsson et al. (2001). In these cases, measurements were available only near the emission centroid altitude, and limited altitudinal coverage was obtained by small changes in the ionospheric interaction altitude during the course of the experiments. In contrast, the side-looking data from HAARP provide for the first time

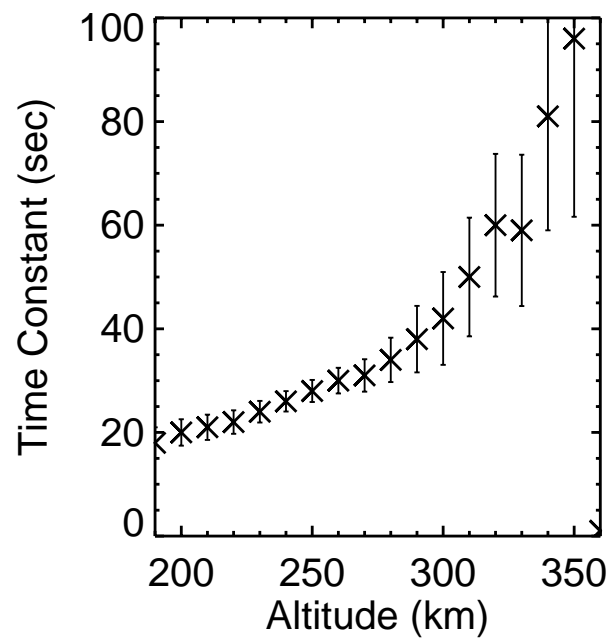

Fig. 6. Effective emission lifetimes for the $O\left({ }^{1} \mathrm{D}\right) 630.0 \mathrm{~nm}$ emission as a function of altitude as determined by fitting the red-line data from Fig. 5 to a model.

measurements of the effective lifetime over a wide range of altitudes independent of the specific interaction altitude.

As an indication of the quality of the model fits we show samples of the data and model comparisons for altitudes of 200, 250, and $300 \mathrm{~km}$ in Fig. 7. Modeled intensities for the best-fit effective lifetimes are shown as thick gray lines, with thin black lines superimposed to show the backgroundsubtracted measured intensities. As the transmitter pulses were only $1 \mathrm{~min}$ in duration throughout this experiment, saturation of intensity was never observed even at the shortest effective lifetimes and lowest altitudes, leaving all pulses with a spike-like appearance. The width of the pulse bases, however, clearly increases with altitude, indicating the larger effective lifetime. At 200 and $250 \mathrm{~km}$ (left and center panels), where the signal intensity is relatively high, the model and data agree very well, in accord with the small error bars provided in Fig. 6. At $300 \mathrm{~km}$ (right panel), however, the signal intensity is much smaller and significant deviations between the model and the data can be seen, especially near the base of the emission spikes where uncertainty in the background removal has the greatest impact. The poorer quality fit at $300 \mathrm{~km}$ altitude is reflected in the much larger error bar in Fig. 6.

Significant changes in relative amplitude between the individual pulses as a function of altitude are also apparent in Fig. 7. At $200 \mathrm{~km}$, the largest pulses are near 03:45 UT, with intensity dropping steadily thereafter, whereas at 250 and $300 \mathrm{~km}$ altitude the largest amplitude pulse is near 04:00 UT. The 4 pulses between $\sim 04: 12$ and 04:25 UT have relatively constant amplitudes at $200 \mathrm{~km}$, and are about half the maximum intensity seen in the experiment at this altitude, but at $250 \mathrm{~km}$ they are well over half the maximum and are close to the amplitude of the other previous pulses at $300 \mathrm{~km}$. Given that the height-integrated amplitudes of the emission 

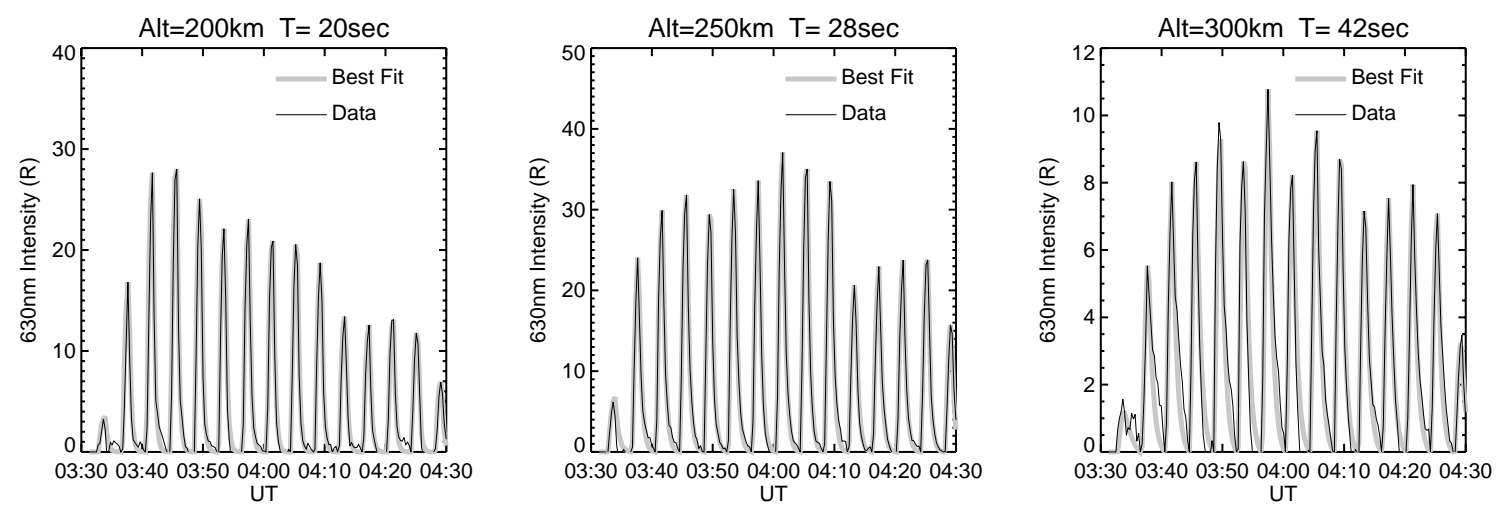

Fig. 7. Sample comparisons of modeled $630.0 \mathrm{~nm}$ emission intensities (thick gray line) and background-subtracted measurements (thin black line) for altitudes of 200, 250, and $300 \mathrm{~km}$ and the effective lifetime time constants plotted in Fig. 6.

intensity for this same experiment, as recorded by the imager at the HAARP site, have recently been used to discriminate between different excitation mechanisms (Kosch et al., 2007b), the large observed variations in intensity of the various pulses with altitude suggest that height-resolved optical emission measurements may provide a means for determining the altitudinal and possibly temporal dependence of the different excitation mechanisms and transport phenomena.

\subsection{Dedicated remote imager network for altitude profiles}

Based on the utility of remote observations, both for altitude profiles and tomography of the optical emissions (see Gustavsson, 2000, and Gustavsson et al., 2001) and to mitigate the risk of adverse weather at the site during optical experiments, a network of several remote optical observatories was proposed for HAARP in 2005. Dubbed HAARPOON (HAARP Optical Observing Network), the design criteria called for a network of 3 or more automated remote stations with at least 2 wavelengths spectral coverage, for a total cost of no more than 1 conventional bare CCD imager. The remote stations were to be able to observe the E-region at 100 $\mathrm{km}$ over HAARP with an elevation angle no less than $30 \mathrm{de}-$ grees, to minimize obstructions from horizon features, haze, and cloud layers. The $\mathrm{F}$ region at $300 \mathrm{~km}$ was required to be observable with an elevation angle of no more than 60 degrees, to ensure an adequate baseline for triangulation of emission altitudes. Additionally, the ability to observe the HAARP beam center azimuth over the full range of beam positions ( $30^{\circ}$ off zenith) was considered highly desirable. Sensitivity adequate to image the weak (a few tens of $R$ at $557.7 \mathrm{~nm}$ ) emissions was of course a fundamental requirement.

Based on these criteria, we produced a design in which $95 \%$ of the hardware budget was allocated to cooled CCD cameras, with 2 cameras at each site operated simultaneously to provide wavelength coverage without the use of failureprone filter wheels or other moving parts. Telecentricity suf-

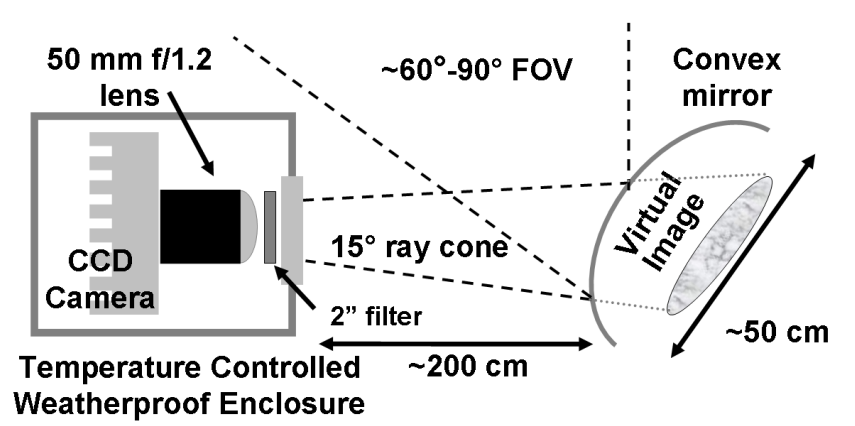

Fig. 8. Schematic of the HAARPOON remote automated imager design concept.

ficient for employment of $2 \mathrm{~nm}$ narrow-band filters $\left( \pm 7.5^{\circ}\right)$ was achieved by utilization of $50 \mathrm{~mm}$ SLR lenses (Nikkor f/1.2) with $1 / 2^{\prime \prime}$ ethernet-based CCD cameras (Apogee Alta E47). This lens and CCD combination provides a $15^{\circ}$ native field of view allowing narrow-band filters to be mounted directly in front of the primary lens. For the sake of economy, we utilized inexpensive $2^{\prime \prime} 10 \mathrm{~nm}$ bandpass filters in stock from Andover Corporation. The desired $60^{\circ}-90^{\circ}$ field of view was achieved at low cost by pointing the $15^{\circ}$ cameras at a large ( $\sim 50 \mathrm{~cm}$ diameter $) 90^{\circ}$ field of view outdoor safety mirror of the type commonly used at blind intersections. The cameras were mounted in an insulated box with a heater, ventilation fan, and thermostat, and aimed through a window at the safety mirror approximately $2 \mathrm{~m}$ distant. The mirror was tilted to eliminate obstruction by the camera enclosure, and the cameras focused on the virtual image of the sky scene located at approximately half the mirror radius behind the mirror. The mirror surface is heated from behind using an electric heating pad to prevent condensation of frost or dew accumulation of snow or ice. A schematic of the HAARPOON design is shown above in Fig. 8.

Although the desired field of view in this mirror-based design can be readily obtained with a small mirror close to the 


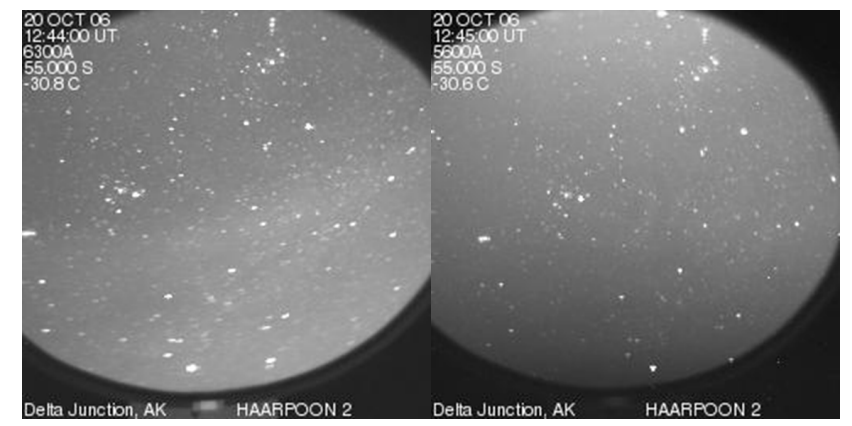

Fig. 9. Star field images from a deployed HAARPOON system in operation in Alaska. The horizon is near the top of the images, with vertical near the bottom. Note the slight deterioration in focus near the bottom of the image on the left, which corresponds to the more distant upper part of the tilted mirror.

camera, the curved and tilted virtual image behind the mirror requires a large depth of field, which depends on the absolute focal length of the lens and the F-number of the lens used. To operate at the lowest possible F-numbers, and therefore the highest sensitivity, depth of field considerations require the system to employ relatively large mirrors far from the camera in order to achieve acceptable focus. For our system, the focal length $l=50 \mathrm{~mm}$, the F-number $f=1.2$, and choosing 2 pixels as an acceptable circle of confusion $c$ on a $13 \mathrm{~mm} \times 13 \mathrm{~mm}$ CCD chip binned down to $256 \times 256$ pixels $(\sim 0.1 \mathrm{~mm} /$ pixel $)$, we get a hyperfocal distance $h \approx l^{2} / f c$ of approximately $20.8 \mathrm{~m}$. The approximate depth of field $d$ can be computed from the hyperfocal distance $h$ of the lens/detector combination and the distance $x$ to the image as $d \approx 2 h x^{2} /\left(h^{2}-x^{2}\right)$. For $x=2 \mathrm{~m}, d$ is approximately $25 \mathrm{~cm}$, just barely adequate to accommodate the tilted virtual image behind the $50 \mathrm{~cm}$ primary mirror selected for the system. As the mirror is moved toward the camera, the depth of field falls off more rapidly than the image shrinks in size and depth (assuming smaller mirrors are used to fill the $15^{\circ}$ field of view), so the $\sim 2 \mathrm{~m}$ mirror distance is a practical minimum for this lens/detector combination, confirmed by experiments with mirrors of various sizes. In practice, spherical aberration and defects in the low-cost mirrors are also non-negligible, so depth of field is not the only limitation.

However, considering the generally diffuse nature of the expected emission structures and the trade-offs between sensitivity, exposure time, and spatial resolution, and cost, we determined that inexpensive abberative optics with twin high-performance $\mathrm{CCD}$ cameras viewing the same mirror were preferable to expensive high-quality optics feeding a single camera or less capable multiple CCD cameras. The star field images in Fig. 9 show the actual performance of the first deployed system. The focus is adequate in the $560 \mathrm{~nm}$ camera, verifying that the depth of field is adequate to accommodate the depth of the virtual image, but the $630 \mathrm{~nm}$ images are somewhat less focused than desired. Although no observations of artificial optical emissions have yet been made in deployed configuration as of this writing, due to construction on the HAARP transmitter array, the sensitivity of the HAARPOON system was confirmed during the March 2006 campaign, when the prototype was operated at the HAARP site in narrow-field mode without the mirrors in place and obtained the $2 \mathrm{~s}$ green line images shown previously in Fig. 4.

\section{Observations of natural optical emissions}

Although a variety of interesting artificial optical phenomena have been observed at HAARP, one of the greatest revelations from improved instrumentation has been the recent realization that the nominally subauroral environment at HAARP is anything but benign, and can have a dramatic impact on HAARP operations.

Various auroral and subauroral phenomena ranging from substorms to SAR arcs were anticipated at HAARP and have been observed on occasion, but one of the most common and disruptive phenomena has been the highly structured hard subauroral precipitation that became apparent when improved imagers were first deployed for the 2004 winter campaigns. This phenomenon, which appears to be a slightly lower latitude manifestation of the "co-rotating aurora" reported by Kubota et al. (2003), has occurred on a number of occasions during heating experiments at HAARP and drastically affected the observed results, particularly by modulating the spatial structure of artificial optical emissions.

As detailed in a separate paper (Pedersen et al., 2007), this hard co-rotating subauroral precipitation as observed from HAARP is commonly found in the dusk and evening sectors, although occasionally remaining well into the morning sector, and is characterized by slow motion, weak intensities at 427.8 and $557.7 \mathrm{~nm}$, and lack of significant red-line emissions at $630.0 \mathrm{~nm}$. It appears in a variety of forms ranging from relatively homogenous bands to widely distributed small-scale $(\sim 10-30 \mathrm{~km})$ curl-like structures dubbed "angels," which appear to be a late phase in the evolution of an instability operating on the more homogenous precipitation regions, especially the poleward edges. Of primary interest for ionospheric heating is the interaction between these regions of natural precipitation and the HF waves generated by the HAARP transmitter.

Figure 10 illustrates the effect of this hard natural precipitation in a dramatic fashion. On 23 March 2004, the HAARP transmitter was operating in a 4 min on-1 min off mode at $2.75 \mathrm{MHz}$ on an otherwise quiet evening with the aurora far to the northeast. A transmitter pulse beginning at 06:35 UT resulted in the familiar enhanced $630.0 \mathrm{~nm}$ red emissions in a small spot at magnetic zenith gradually tapering off away from the field aligned position at 06:38 UT. At about 06:45, a faint band a few tens of $\mathrm{R}$ in intensity above the sky background appeared overhead in the $557.7 \mathrm{~nm}$ green-line 


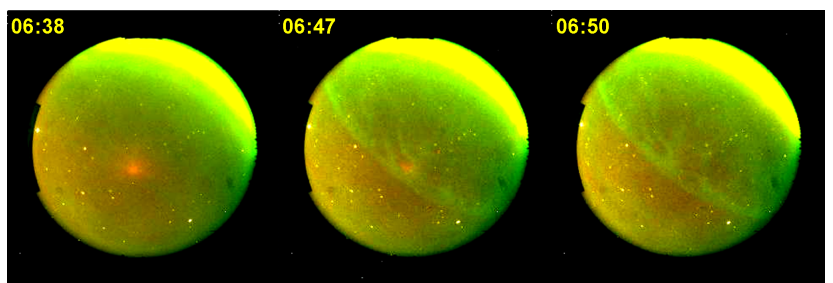

Fig. 10. Composite color $(630.0,557.7$, and $427.8 \mathrm{~nm})$ image of Fregion heating (red spot near center) blocked by hard precipitation (irregular green band) that appeared overhead during an experiment on 23 March 2004. Saturated colors represent an intensity of $200 \mathrm{R}$. Note the red emissions continuing through a gap in the precipitation in the image for 06:47 UT.

images. In the image at 06:47 UT, the red emissions return, but are now limited to a small region between irregular structures on the poleward edge of the green band. The narrowed spot moves slightly with the precipitation structure for the next frame (not shown) but by 06:50 UT the artificial emissions are completely blocked by a wider part of the precipitation band.

Figure 11 shows composite color (630.0 and $557.7 \mathrm{~nm}$ ) images for another heating experiment carried out in the presence of hard subauroral precipitation. In this case the precipitation appears as widely distributed irregular structure slowly drifting toward the magnetic south-southwest in the $557.7 \mathrm{~nm}$ green-line images. The transmitter was operating at $2.83 \mathrm{MHz}$ in a $2 \mathrm{~min}$ on 2 min off mode. In the image for 06:11 UT the artificial emissions (red) are clearly seen illuminating the region near the magnetic zenith (marked with a white plus sign) through a large hole in the natural precipitation. A few minutes later, at 06:15, the precipitation has drifted over the magnetic zenith, but the artificial emissions are now seen only in the gap to the south, where the intensity is significantly lower than in the magnetic zenith. By 06:23, the next gap in the precipitation approaches magnetic zenith, and fills with artificial emissions while still some distance away. This same effect repeats numerous times during the course of the experiment, with the red line artificial emissions suppressed in areas where green line images show hard precipitation.

These image sequences clearly demonstrate that hard subauroral precipitation spatially modulates the excitation of artificial optical emissions. There are three potential mechanisms that could explain this effect: 1) F-region densities in flux tubes containing the precipitation are significantly different from the ionosphere outside the precipitation and either do not support emission-producing resonances or reflect or refract the transmitter beam away from these flux tubes; 2 ) Eregion densities created by the precipitation reflect or refract $\mathrm{HF}$ waves away from the F-region within the precipitation regions; or 3) enhanced $\mathrm{D}$ region density created by the precipitating particles absorbs HF waves before they reach the $\mathrm{E}$

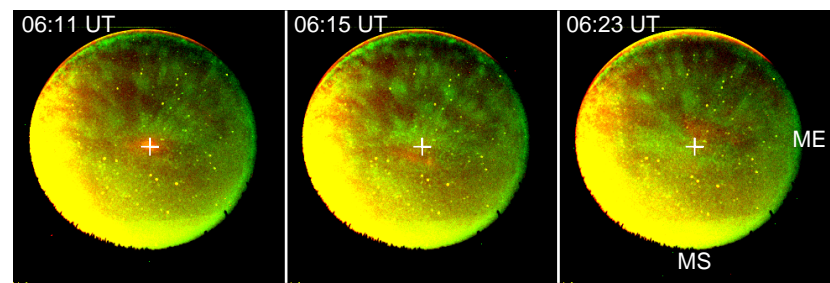

Fig. 11. Widespread highly structured subauroral precipitation (visible as green in these composite color images) drifting slowly across the field of view during a heating experiment on 26 March 2006. The artificial optical emissions (red) appear only in the gaps in the natural precipitation.

and $\mathrm{F}$ regions. Mechanism 1) would be expected to result in multiple traces on ionograms, as some frequencies from the wide range emitted by the ionosonde would be expected to successfully penetrate the precipitation flux tubes even if the heater frequency was prevented from reaching that volume. Mechanism 2) can clearly operate in sporadic and auroral E layers, but, as with the first mechanism, E-region ionization sufficient to disrupt propagation to the F region should produce detectable signatures in ionograms.

Under mechanism 3), enhanced $D$ region densities in the precipitation region would absorb the HF waves from both the transmitter and the ionosonde, restricting optical emissions and $\mathrm{E}$ and $\mathrm{F}$ region ionosonde echoes to flux tubes devoid of precipitation. For a typical broad ionosonde beam and partial precipitation coverage, no obvious signature would be apparent in the ionograms, apart from a possibly smaller number of echoes, as returns from precipitationfree flux tubes within the ionosonde beam would remain present. Enhanced E region ionization created by the precipitation may or may not be present above the absorption layer in the absorption scenario, depending on the specific energies and fluxes of the precipitating particles, but would not be observable except in oblique traces where the HF could reach the $\mathrm{E}$ layer through localized holes in the absorbing $\mathrm{D}$ layer.

The absorption-based scenario would appear to fit best with actual observations from HAARP. For the case shown in Fig. 10, ionosonde echoes faded away as the precipitation band appeared (Pedersen et al., 2007), suggesting that absorption was indeed associated with the precipitation. On the other hand, full ionograms were obtained throughout the 26 March 2006 experiment, but did not show any detectable E region or obvious multiple traces in the F region. Some other cases of hard subauroral precipitation from HAARP do have detectable E layers, but primarily on oblique traces, consistent with what would be expected if $\mathrm{D}$ region absorption were present below the layer allowing the $\mathrm{E}$ region to be observed only from the side after penetrating through holes in the absorption layer. 
One additional test that could be performed in the future would be to examine spatial maps of ionosonde echoes to determine whether they are restricted to precipitation-free areas as the artificial optical emissions are, or have some spatial distribution unrelated to the precipitation pattern. Another test would be to attempt to generate artificial emissions at larger angles from the magnetic zenith so the large difference in altitude between the $\mathrm{D}$ region absorption and the F region emissions would produce a clearly detectable horizontal displacement in the pattern between the natural optical emissions indicating the location of the precipitation and the artificial optical emissions modulated by $\mathrm{D}$ layer absorption.

\section{Summary and conclusions}

A number of interesting optical observations have been made at the HAARP facility since 2004 but prior to expansion of the transmitter array to its full $3.6 \mathrm{MW}$ power level.

Quantifiable levels of optical emission from beam swinging experiments across and along the magnetic meridian have shown steep cut-offs in emission production by $15^{\circ}$ zenith angle in the magnetic east, west, and north, while in the magnetic south higher production is seen from the vertical through points south of magnetic zenith. It has yet to be determined whether these sharp gradients result from propagation conditions or the actual excitation mechanisms.

High temporal resolution observations of short transmitter pulses and pulse leading edges at $557.7 \mathrm{~nm}$ show preferential excitation of structures with scale sizes of 5-10 km in the first few seconds of heating which diffuse out somewhat over time during extended heating period but remain relatively unchanged when excited only by short pulses.

Side-looking altitude profiles from remote observations of HAARP artificial optical emissions span a range of $200 \mathrm{~km}$ and allow excitation mechanisms and the effective lifetime of the $\mathrm{O}\left({ }^{1} \mathrm{D}\right)$ state to be examined over a wide range of altitudes. Our estimates of the effective lifetime of $\mathrm{O}\left({ }^{1} \mathrm{D}\right)$ cover an unprecedented altitude range from 190 to $350 \mathrm{~km}$ and time constants from $<20$ to $>90 \mathrm{~s}$, and are expected to be a key data source for determining neutral atmospheric densities. The new HAARPOON remote imager network, which utilizes an innovative low-cost mirror-based design, will provide automated remote observations to allow routine determination of these important parameters.

Improved optical diagnostics at HAARP have allowed the detection of faint $557.7 \mathrm{~nm}$ emissions from highly structured hard sub-auroral particle precipitation, which we have shown is capable of dramatically modulating the spatial structure of ionospheric heating and artificial optical emissions by suppressing heating effects in regions occupied by the precipitation. The details of this common but previously unrecognized effect are consistent with localized absorption of the HF waves by D-layer ionization enhanced by the particle pre- cipitation, although we have suggested additional tests which can be performed to verify the action of this mechanism.

With the HAARP transmitter now upgraded to full 3.6 MW capacity we expect the facility to produce additional new and interesting optical effects, in addition to allowing rarely seen effects to be reproduced on a regular basis.

Acknowledgements. HAARP is a Department of Defense project operated jointly by the U.S. Air Force and U.S. Navy. Research at AFRL was carried out under AFOSR task 2311AS. We thank M. McCarrick for providing the beam power contours used in Figs. 1 and 2.

Topical Editor U.-P. Hoppe thanks M. Rietveld and another anonymous referee for their help in evaluating this paper.

\section{References}

Bernhardt, P. A., Tepley, C. A., and Duncan, L. M.: Airglow enhancements associated with plasma cavities formed during ionospheric heating experiments, J. Geophys. Res., 94(A7), 90719092, 1989.

Bernhardt, P. A., Wong, M., Huba, J. D., Fejer, B. G., Wagner, L. S., Goldstein, J. A., Selcher, C. A., Frolov, V. L., and Sergeev, E. N.: Optical remote sensing of the thermosphere with HF pumped artificial airglow, J. Geophys. Res., 105(A5), 10 657-10 671, 2000.

Brandstrom, B.: The Auroral Large Imaging System - Design, operation and scientific results, IRF Scientific Report 279, Swedish Institute of Space Physics, Kiruna, Sweden, 2003.

Brandstrom, B. U. E., Leyser, T. B., Steen, A., Rietveld, M. T., Gustavsson, B., Aso, T., and Ejiri, M.: Unambiguous evidence of HF pump-enhanced airglow at auroral latitudes, Geophys. Res. Lett., 26, 2561-3564, 1999.

Djuth, F. T., Pedersen, T. R., Gerken, E. A., Bernhardt, P. A., Selcher, C. A., Bristow, W. A., and Kosch, M. J.: Ionospheric modification at twice the electron cyclotron frequency, Phys. Rev. Lett., 94, 125 001, doi:10.1103/PhysRevLett.94.125001, 2005.

Gustavsson, B., Sergienko, T., Rietveld, M. T., Honary, F., Steen, A., Brandstrom, B. U. E., Leyser, T. B., Aruliah, A. L., Aso, T., Ejiri, M., and Marple, S.: First tomographic estimate of volume distribution of HF-pump enhanced airglow emissions, J. Geophys. Res., 106(A12), 29 105-29 123, 2001.

Gustavsson, B.: Three dimensional imaging of auroral and airglow, Ph.D thesis, Swedish Institute of Space Physics, Kiruna, Sweden, 2000.

Kosch, M. J., Rietveld, M. T., Kavanagh, A. J., Davis, C., Yeoman, T. K., Honary, F., and Hagfors, T.: High-latitude pumpinduced optical emissions for frequencies close to the third electron gyro-harmonic, Geophys. Res. Lett., 29(23), 2112, doi:10.1029/2002GL015744, 2002.

Kosch, M. J., Pedersen, T., Hughes, J., Marshall, R., Gerken, E., Senior, A., Sentman, D., McCarrick, M., and Djuth, F. T.: Artificial optical emissions at HAARP for pump frequencies near the third and second electron gyro-harmonic, Ann. Geophys., 23, 15851592, 2005, http://www.ann-geophys.net/23/1585/2005/.

Kosch, M. J., Pedersen, T., Mishin, E., Starks, M., Gerken, E., Sentman, D., Oyama, S., and Watkins, B.: Temporal evolution 
of pump beam self-focusing at HAARP, J. Geophys. Res., 112, A06325, doi:10.1029/2006JA012146, 2007a.

Kosch, M. J., Pedersen, T., Mishin, E., Oyama, S., Hughes, J., Senior, A., Watkins, B., and Bristow, B.: Coordinated optical and radar observations of ionospheric pumping for a frequency pass through the second electron gyro-harmonic at HAARP, J. Geophys. Res., 112, A06325, doi:10.1029/2006JA012146, $2007 \mathrm{~b}$.

Kubota, M., Nagatsuma, T., and Murayama, Y.: Evening co-rotating patches: A new type of aurora observed by high-sensitivity all-sky cameras in Alaska, Geophys. Res. Lett., 30(12), 1612, doi:10.1029/2002GL016652, 2003.
Pedersen, T. R. and Carlson Jr., H. C.: First observations of HF heater-produced airglow at the High Frequency Active Auroral Research Program facility: Thermal excitation and spatial structuring, Radio Sci., 36, 1013-1026, 2001.

Pedersen, T. R., Mishin, E., and Oksavik, K.: Observations of structured optical emissions and particle precipitation equatorward of the traditional auroral oval, J. Geophys. Res., accepted, 2007.

Pedersen, T. R., McCarrick, M., Gerken, E., Selcher, C., Sentman, D., Carlson, H. C., and Gurevich, A.: Magnetic zenith enhancement of HF radio-induced airglow production at HAARP, Geophys. Res. Lett., 30, 1169, doi:10.1029/2002GL016096, 2003. 